\title{
The management of acute coronary syndromes in patients presenting without persistent ST-segment elevation: key points from the ESC 2020 Clinical Practice Guidelines for the general and emergency physician
}

\author{
Authors: Ramesh Nadarajah ${ }^{A}$ and Chris Gale ${ }^{B}$
}

\begin{abstract}
There have been significant advances in the diagnosis and management of non-ST-segment elevation myocardial infarction over recent years, which has been reflected in an international decline in mortality rates. This article provides an overview of the 2020 European Society of Cardiology Clinical Practice Guidelines for the topic, concentrating on areas relevant to the general or emergency physician. The recommendations and underlying evidence basis are analysed in three key areas: diagnosis (the recommendation to use high sensitivity troponin and how to apply it), pathways (the recommendation to facilitate early invasive coronary angiography to improve outcomes and shorten hospital stays) and treatment (a paradigm shift in the use of early intensive platelet inhibition). Gaps in the evidence base are highlighted, including the optimal management strategy for older people and the antiplatelet regime to consider when angiography may be delayed.
\end{abstract}

KEYWORDS: acute coronary syndrome, NSTEMI, myocardial infarction, ESC clinical practice guideline, coronary angiography

DOI: $10.7861 /$ clinmed.2020-0879

\section{Introduction}

Non-ST-segment elevation myocardial infarction (NSTEMI) is the most prevalent acute coronary syndrome (ACS) presentation in the UK. Data from the UK Myocardial Infarction National Audit Project (MINAP) found that between April 2017 and March 2018 there were 56,493 admissions nationally for NSTEMI, an increase of $5 \%$ from the previous year. ' Over recent years, however, there have been substantial therapeutic advances in how we care for people with NSTEMI, and this has been reflected in an

Authors: ${ }^{A}$ British Heart Foundation clinical training fellow and cardiology specialty registrar, Leeds Institute of Cardiovascular and Metabolic Medicine, Leeds, UK and Leeds General Infirmary, Leeds, UK; ${ }^{B}$ professor of cardiovascular medicine and honorary consultant cardiologist, Leeds Institute of Cardiovascular and Metabolic Medicine, Leeds, UK and Leeds General Infirmary, Leeds, UK international decline in mortality rates. ${ }^{2,3}$ In September 2020, the European Society of Cardiology (ESC) published updated Clinical Practice Guidelines for the management of ACS in patients presenting without persistent ST-segment elevation, ${ }^{4} 5$ years after the last iteration.

The guidelines stipulate a number of updated recommendations (supplementary material S1). The strength of a recommendation and level of evidence used to justify it are weighted and graded according to predefined scales (Table 1). This focused review provides learning points derived from the guidelines in areas relevant to general and emergency physicians, including diagnosis (recommendation to use high sensitivity troponin), pathways (recommendation to proceed to invasive coronary angiography [ICA] within 24 hours if invasive strategy is deemed suitable), and treatment (review of the merits of early prescription of $\mathrm{P}_{2} \mathrm{Y}_{12}$ receptor inhibitors). In line with the guidelines, acute myocardial infarction (AMI) is defined according to the 4 th universal definition of myocardial infarction (Table 2 ). ${ }^{5}$

\section{Diagnosis \\ Background}

Cardiac troponins are the most sensitive and specific markers of cardiomyocyte injury, superseding older biomarkers such as creatinine kinase (CK), its myocardial band isoenzyme (CK-MB) and myoglobin. They rise quickly (within 1 hour of symptom onset) and stay elevated for several days. Refinement to produce high sensitivity troponin (hs-cTn) assays has led to an increased detection of previously undetectable cardiomyocyte injury and thus increased diagnostic accuracy at identical low cost to less sensitive versions. ${ }^{6}$

\section{Recommendation}

It is recommended to use hs-cTn assay as part of a ' 0 hour $/ 1$ hour' or ' 0 hour $/ 2$ hour' rule-in and rule-out algorithm (class of recommendation I, level of evidence $B$ ).

\section{Rationale}

Due to the higher sensitivity of hs-cTn, the interval between the first and second troponin measurement may be shortened. 
Table 1. Definitions of class of recommendation and supporting level of evidence used in European Society of Cardiology (ESC) guidelines ${ }^{4}$

Recommendations

Class of Recommendation

Class I

Class II

Class IIa

Class IIb

Class III

\section{Levels of evidence}

Levels

A

$B$

C

\section{Definition}

Evidence and/or general agreement that a given treatment or procedure is beneficial, useful, effective

Conflicting evidence and/or a divergence of opinion about the usefulness/ efficacy of the given treatment or procedure

Weight of evidence/opinion is in favour of usefulness/efficacy

Usefulness/efficacy is less well established by evidence/opinion

Evidence or general agreement that the given treatment or procedure is not useful/effective and in some cases may be harmful

\section{Definition}

Data derived from multiple randomised clinical trials or meta-analyses

Data derived from a single randomised clinical trial or large non-randomised studies

Consensus of opinion of the experts and/or small studies, retrospective studies, registries
Optimal thresholds for each available assay have been defined for 'very low', 'low', 'high' and 'delta change' to allow a negative predictive value (NPV) of $99 \%$ and minimal positive predictive value (PPV) of $70 \%{ }^{4}$ The current recommendation is to use these assays in the emergency department as part of a rapid rule-in/ rule-out algorithm - either '0 hour/1 hour' (blood drawn for hs-cTn at 0 hours and 1 hour of attendance) or ' 0 hour $/ 2$ hours' (blood drawn for hs-cTn at 0 hours and 2 hours of attendance) depending on the specific hs-cTn assay available at a centre (Fig 1).

In the recent multi-centre RAPID-TnT randomised controlled trial (RCT), the 0 hour/ 1 hour protocol was shown to be non-inferior to the standard repeat troponin assessment at 3 hours with a significantly higher rate of discharge, shorter stay in the emergency department, lower referral for further functional cardiac testing and an NPV for 30-day death or myocardial infarction of $99.6 \%{ }^{7}$ With ever-increasing demand on acute

Table 2. Definition of acute myocardial infarction as per the fourth universal definition of myocardial infarction (European Society of Cardiology, 2018) ${ }^{5}$

\section{Acute myocardial infarction:}

Detection of an increase and/or decrease of a cardiac biomarker with at least one value above the 99th percentile of the upper reference limit and at least one of:

$>$ symptoms of myocardial ischaemia

$>$ new ischaemic ECG changes or development of pathological Q waves

> imaging evidence of a loss of viable myocardium or new regional wall motion abnormality in pattern consistent with ischaemic aetiology

> intracoronary thrombus detected on angiography or autopsy.

Non-ST-segment elevation myocardial infarction:

The criteria for AMI met without persistent ST-segment elevation ( $>20$ minutes) or new left bundle branch block

\section{Wording used}

It is recommended or indicated

\section{Should be considered}

May be considered

It is not recommended 
Box 1. Common conditions other than acute

myocardial infarction associated with cardiomyocyte injury and cardiac troponin elevation

\section{Cardiovascular conditions}

Tachyarrhythmias

Heart failure

Hypertensive crisis

Valvular heart disease

Myocarditis

Takutsubo syndrome

\section{Non-cardiovascular conditions}

Sepsis

Aortic dissection

Pulmonary embolism

Stroke or subarachnoid haemorrhage

Renal dysfunction with associated cardiac disease

Extreme endurance efforts

Burns

(Box 1), and outcomes for some of these will be adversely affected by the prescription of antiplatelet agents. In addition, age (comparing very old with very young), chronic kidney disease (comparing very high with very low estimated glomerular filtration rate [eGFR]), and time from chest pain onset may cause up to a $300 \%$ difference in troponin values, while sex can cause up to a $40 \%$ difference. ${ }^{8}$

\section{Gaps in the evidence}

Currently, a uniform cut-off hs-cTn level is used for the early diagnosis of AMI despite the aforementioned variables that may affect its concentration. The development of an information technology tool that can incorporate all four variables to arbitrate appropriate cut-offs for individual patients for the diagnosis of AMI may be of help in routine clinical practice. In addition, for the cohort of patients whose two hs-cTn concentrations are in an intermediate range between rule-in and rule-out, the optimum diagnostic strategy is uncertain, with options including the use of additional biomarkers or non-invasive imaging. Studies that randomise patients to diagnostic pathways with or without the such tests in addition to usual care could help clarify the most efficient and cost-effective diagnostic strategy.

\section{Pathways}

\section{Background}

Invasive coronary angiography helps clarify whether chest pain may be due to myocardial ischaemia secondary to a culprit lesion in the epicardial coronary arteries. Obstructive coronary lesions can then be treated by percutaneous coronary intervention (PCI) or coronary artery bypass graft (CABG) surgery depending on their morphology and the patient's clinical characteristics. Although all invasive procedures have inherent risk, these have been somewhat mitigated by advances including radial access and modern drug-eluting stents. Many RCTs have compared a routine invasive strategy with a selective invasive strategy (where invasive coronary angiography would only be performed in the context of recurrent symptoms or evidence of obstructive coronary artery disease from non-invasive tests). Meta-analyses of data from these RCTs have shown a reduction in risk of death or myocardial infarction from an invasive strategy in NSTEMI, especially for patients of high ischaemic risk. ${ }^{9}$ Thus, the guidelines recommend pursuing an invasive coronary strategy within specific time bands based on baseline patient risk.

\section{Recommendation}

Very high risk - immediate invasive strategy ( $<2$ hours) [akin to primary PCI (PPCI)] if at least one of the following present:(class of recommendation $\mathrm{I}$, level of evidence $\mathrm{C}$ )

$>$ haemodynamic instability

$>$ life-threatening arrhythmias

> mechanical complication eg severe mitral regurgitation

$>$ acute heart failure

$>$ cardiogenic shock

$>$ recurrent or refractory chest pain

$>$ ST-segment depression $>1 \mathrm{~mm}$ in 6 leads plus ST-segment elevation in AVR and/or V1.

High risk - early invasive strategy ( $<24$ hours) if at least one of the following present: (class of recommendation I, level of evidence A)

$>$ dynamic rise or fall in troponin with at least 1 value above the 99th percentile of the upper reference limit (NSTEMI)

$>$ GRACE risk score $>140$

$>$ dynamic new or presumably new contiguous ST/T-segment changes

$>$ transient ST-segment elevation

$>$ resuscitated cardiac arrest without ST-segment elevation or cardiogenic shock.

\section{Rationale}

The recommendation for invasive coronary angiography within 24 hours for any patient with a diagnosis of NSTEMI is more aggressive than the recent National Institute for Health and Care Excellence (NICE) quality statement, which recommends invasive coronary angiography within 72 hours of admission. ${ }^{10}$ The two largest studies assessing the benefit of invasive coronary angiography within 24 hours ('early'), TIMACS (Timing of Intervention in Patients with Acute Coronary Syndromes) and VERDICT (Very Early vs Deferred Invasive evaluation using Computerised Tomography), showed a benefit with the 'early' invasive strategy for composite ischaemic endpoints among those with a GRACE risk score $>140$ (the preferred risk scoring system for mortality following ACS). ${ }^{11,12}$ Furthermore, a meta-analysis found lower mortality rates in the 'early' intervention group when patients had at least one of the following: elevated cardiac biomarkers at baseline (diagnosis of NSTEMI), diabetes mellitus, a GRACE risk score $>140$, age $>75$ years. ${ }^{13}$ From a health economics standpoint, another meta-analysis has shown that 'early' invasive coronary angiography leads to shorter in-hospital stays and a UK analysis showed that such a strategy is cost-effective in high-risk patients. ${ }^{14,15}$

\section{Gaps in the evidence}

The recommendation for immediate invasive coronary angiography in patients with a 'very high' risk characteristic is based on the adverse short- and long-term prognosis of this cohort if left untreated. It must be remembered, however, that such patients are usually excluded from RCTs, and so the low 
level of evidence attributed to this recommendation reflects a gap in robust data, which is being addressed by the British Heart Foundation funded RapidNSTEMI (Very Early Versus Delayed Angiography $+/$ - Intervention on Outcomes in Patients with NSTEMI) trial. ${ }^{16}$

Moreover, under-representation of older patients in landmark RCTs of PCI has led to uncertainty as to whether an invasive coronary strategy confers benefit in this group. Recently, a small open-label RCT suggested a reduction in major adverse cardiovascular events (mainly driven by the prevention of further myocardial infarction or urgent revascularisation), without an increase in bleeding complications, from an early invasive coronary strategy in patients $>80$ years of age. ${ }^{17}$ Thus, the updated recommendation is to employ the same interventional strategies in older patients as younger patients. Separately, frail patients with NSTEMI have longer hospital stays, higher risk of death and major bleeding..$^{18}$ The lack of robust evidence in this group means clinicians may have to make caseby-case decisions on whether to proceed with an invasive coronary strategy by assessing the risks of future cardiovascular events versus peri-procedural complications, but also life expectancy, comorbidities, quality of life and patient preferences. The ongoing British Heart Foundation funded SENIOR-RITA (older patients with non-ST SEgmeNt elevatIOn myocaRdial infarction Randomised Interventional TreAtment) trial comparing invasive versus conservative strategies for patients $>75$ years of age will also address frailty status, and should help provide a stronger evidence basis. ${ }^{19}$

\section{Treatment}

\section{Background}

The previous recommended treatment for NSTEMI comprised routine use of dual antiplatelet treatment (DAPT) and anticoagulant (usually fondaparinux at $2.5 \mathrm{mg}$ subcutaneous/day) from the time of diagnosis. The favoured antiplatelet regime was the combination of aspirin (300 mg loading dose then $75 \mathrm{mg} /$ day) alongside ticagrelor (180 mg loading dose then $90 \mathrm{mg}$ twice daily). ${ }^{20}$

Aspirin irreversibly inactivates cyclooxygenase activity and suppresses thromboxane $A_{2}$ production throughout the platelet lifespan. Meta-analysis of data from the pre-PCI era has shown a $46 \%$ reduction for major vascular events with aspirin treatment for ACS. ${ }^{21}$ The addition of a $\mathrm{P} 2 \mathrm{Y}_{12}$ receptor inhibitor at diagnosis to inhibit adenosine diphosphate (ADP)-induced platelet aggregation was initially shown to reduce ischaemic events in ACS in patients presenting without persistent ST-segment elevation with clopidogrel in the CURE trial (Clopidogrel in Unstable Angina to Prevent Recurrent Events).22 This was superseded by ticagrelor after the PLATO (PLATelet inhibition and patient Outcomes) trial found its greater potency led to a further reduction in ischaemic events without an increase in fatal or life-threatening bleeds, irrespective of receipt of PCI. ${ }^{23}$ Prasugrel (another potent P2Y receptor inhibitor) also led to a reduction in ischaemic events when compared with clopidogrel, but with more frequent severe bleeding complications. ${ }^{24}$

\section{Recommendation}

> Prasugrel should be considered in preference to ticagrelor for patients who proceed to PCI (class of recommendation IIa, level of evidence B).

> If an early invasive management strategy is planned it is not recommended to routinely administer pre-treatment with a
$\mathrm{P} 2 \mathrm{Y}_{12}$ receptor inhibitor (class of recommendation III, level of evidence A).

> If an early invasive management strategy is not planned then administration of pre-treatment with a $\mathrm{P} 2 \mathrm{Y}_{12}$ receptor inhibitor may be considered in the absence of high bleeding risk (class of recommendation IIb, level of evidence $\mathrm{C}$ ).

\section{Rationale}

Contemporary data challenge the concept of early intense platelet inhibition with $\mathrm{P}_{2} \mathrm{Y}_{12}$ receptor inhibitors for patients who are planned for an invasive strategy ('pre-treatment'). Observational data from a large Swedish dataset showed that pre-treatment was associated with a significantly increased risk of bleeding events without an improvement in ischaemic outcomes. ${ }^{25}$ Of course, pre-treatment may be associated with patient harm should the diagnosis not be AMI but, for example, aortic dissection or subarachnoid haemorrhage. The more rapid onset of action after loading doses of prasugrel and ticagrelor (30 minutes) also makes it viable to only administer them during invasive coronary angiography once the coronary anatomy has been delineated and it is decided to proceed to PCI.

The ISAR-REACT (Intracoronary stenting and antithrombotic regimen: Rapid Early Action for Coronary Treatment) 5 trial compared the strategy of pre-treatment with ticagrelor to deferred loading with prasugrel (60 mg then $10 \mathrm{mg} /$ day) at invasive coronary angiography once the decision was made for PCI. In a trial with a high proportion of patients treated with PCI $(84 \%)$, the prasugrel arm showed a significantly lower composite endpoint of all-cause death, myocardial infarction and stroke at 1 year (primarily driven by a reduced incidence of myocardial infarction) without an increased incidence in major bleeding events. ${ }^{26}$ The 2020 ESC guidelines therefore no longer recommend pre-treatment with a P2 $Y_{12}$ receptor inhibitor if an 'early' invasive management strategy is planned, and recommend prasugrel loading when PCI has been decided upon.

For patients who will receive 'delayed' invasive coronary angiography, the prescription of $\mathrm{P} 2 \mathrm{Y}_{12}$ receptor inhibitors should no longer be routine, but carefully considered after factoring in the patient's bleeding risk. The bleeding risk may be estimated from scoring systems such as CRUSADE (Can Rapid Risk stratification of Unstable angina patients Suppress Adverse outcomes with Early Implementation of the ACC/ AHA guidelines) or by identifying major and minor criteria according to ARC-HBR (Academic Research Consortium for High Bleeding Risk). ${ }^{27,28}$ Among patients for whom conservative management is planned, DAPT (preferably with ticagrelor) is still recommended at the time of diagnosis and fondaparinux is still recommended for both a conservative strategy and when invasive coronary angiography is not possible within 24 hours. $^{4}$

The long-term combination and duration of antiplatelets following NSTEMI is at the discretion of the treating interventionalist and is dependent on ischaemic risk, bleeding risk and whether there is a co-existent indication for oral anticoagulation. The other components of the long-term management of NSTEMI have seen updates from the most recent guidelines on hypertension, diabetes mellitus and hypercholesterolaemia (supplementary material S2).

\section{Gaps in the evidence}

The most recent evaluation of UK clinical practice found 19.1\% of patients with NSTEMI received invasive coronary angiography within 24 hours. 1 As such, most patients will require careful consideration of their bleeding risk before the prescription of 
a P2Y ${ }_{12}$ receptor inhibitor. Data have shown that prasugrel's predominant benefit is when PCI will definitely occur, whereas early prescription is associated with bleeding complications. Therefore RCTs which compare pre-treatment with ticagrelor versus placebo against loading at the time of invasive coronary angiography, within a timeframe of 72 hours of presentation, could help clarify the optimal antiplatelet regime when 'early' invasive coronary angiography is not possible.

\section{Conclusion}

Over the last 25 years, there has been substantial progress in the management of ACS in patients presenting without persistent STsegment elevation, driven by major advances in invasive coronary techniques, new pharmacotherapies and biochemical assays. The 2020 ESC guidelines emphasise the importance of a personalised approach to care which involves the use of these innovations. This includes more sensitive detection of NSTEMI, a more precise approach to antiplatelet therapy to reduce bleeding complications, and the potential benefit from an expedient invasive coronary strategy for higher-risk patients. Even so, there are important gaps in the knowledge base, which may be clarified by robust evidence from RCTs, such as the optimal treatment strategy for older people and the safest antiplatelet regimen when 'early' invasive coronary angiography is not possible.

\section{Supplementary material}

Additional supplementary material may be found in the online version of this article at www.rcpjournals.org/clinmedicine: S1 - Key recommendations from the 2020 ESC guidelines for the management of non-ST-segment elevation myocardial infarction S2 - Long-term management of non-ST-segment elevation myocardial infarction

\section{References}

1 National Institute for Cardiovascular Outcomes Research. Myocardial Ischaemia National Audit Project: 2019 summary report (2017/18 data). NICOR, 2019. Available from www.hqip.org.uk/resource/ myocardial-ischaemia-national-audit-project-minap-2019-summaryreport/.

2 Hall M, Dondo TB, Yan AT et al. Association of clinical factors and therapeutic strategies with improvements in survival following non-ST-elevation myocardial infarction, 2003-2013. JAMA 2016;316:1073-82.

3 Simonsson M, Wallentin L, Alfredsson ] et al. Temporal trends in bleeding events in acute myocardial infarction: insights from the SWEDEHEART registry. Eur Heart J 2020;41:833-43.

4 Collet J-P, Thiele H, Barbato E et al. 2020 ESC Guidelines for the management of acute coronary syndromes in patients presenting without persistent ST-segment elevation. Eur Heart J 2020, in press (DOI: 10.1093/eurheartj/ehaa575).

5 Thygesen K, Alpert JS, Jaffe AS et al. Fourth universal definition of myocardial infarction (2018). Eur Heart ] 2019;40:237-69.

6 Shah AS V, Anand A, Strachan FE et al. High-sensitivity troponin in the evaluation of patients with suspected acute coronary syndrome: a stepped-wedge, cluster-randomised controlled trial. Lancet 2018;392:919-28.

7 Chew DP, Lambrakis K, Blyth A et al. A randomized trial of a 1-hour troponin T protocol in suspected acute coronary syndromes: the rapid assessment of possible acute coronary syndrome in the emergency department with high-sensitivity troponin T Study (RAPID-TnT). Circulation 2019;140:1543-56.
8 Miller-Hodges E, Anand A, Shah AS et al. High-sensitivity cardiac troponin and the risk stratification of patients with renal impairment presenting with suspected acute coronary syndrome. Circulation 2018;137:425-35.

9 Fox KAA, Clayton TC, Damman P et al. Long-term outcome of a routine versus selective invasive strategy in patients with non-STsegment elevation acute coronary syndrome a meta-analysis of individual patient data. J Am Coll Cardiol 2010;55:2435-45.

10 National Institute of Health and Care Excellence. Quality statement 3: Coronary angiography and PCI within 72 hours for NSTEMI or unstable angina. In Acute coronary syndromes in adults. Quality standard [QS68]. NICE, 2014. www.nice.org.uk/guidance/qs68/ chapter/Quality-statement-3-Coronary-angiography-and-PCIwithin-72-hours-for-NSTEMI-or-unstable-angina.

11 Mehta SR, Granger CB, Boden WE et al. Early versus delayed invasive intervention in acute coronary syndromes. $N$ Engl J Med 2009;360:2165-75.

12 Kofoed KF, Kelbæk H, Hansen PR et al. Early versus standard care invasive examination and treatment of patients with nonst-segment elevation acute coronary syndrome. Circulation 2018:138:2741-50.

13 Jobs A, Mehta SR, Montalescot G et al. Optimal timing of an invasive strategy in patients with non-ST-elevation acute coronary syndrome: a meta-analysis of randomised trials. Lancet 2017;390:737-46.

14 Bonello L, Laine M, Puymirat E et al. Timing of coronary invasive strategy in non-ST-segment elevation acute coronary syndromes and clinical outcomes: an updated meta-analysis. JACC Cardiovasc Interv 2016;9:2267-76.

15 Henriksson M, Epstein DM, Palmer S] et al. The cost-effectiveness of an early interventional strategy in non-ST-elevation acute coronary syndrome based on the RITA 3 trial. Heart 2008;94:717-23.

16 Kite TA, Gersh B], Gershlick AH. Spotlight on N-STEMI ACS: getting the right patients the right treatment, and at the right time. EuroIntervention 2019;15:e1041-5.

17 Tegn N, Abdelnoor M, Aaberge L et al. Invasive versus conservative strategy in patients aged 80 years or older with non-ST-elevation myocardial infarction or unstable angina pectoris (After Eighty study): an open-label randomised controlled trial. Lancet 2016;387: 1057-65.

18 Ekerstad N, Swahn E, Janzon M et al. Frailty is independently associated with short-term outcomes for elderly patients with non-ST-segment elevation myocardial infarction. Circulation 2011;124:2397-404.

19 Chan D, Lawson L, Kunadian V. Management of older patients presenting with non-ST-elevation acute coronary syndrome. EuroIntervention 2018;14:e258-60.

20 Roffi M, Patrono C, Collet J-P et al. 2015 ESC Guidelines for the management of acute coronary syndromes in patients presenting without persistent ST-segment elevation. Eur Heart J 2016;37:267-315.

21 Antithrombotic Trialists' Collaboration. Collaborative meta-analysis of randomised trials of antiplatelet therapy for prevention of death, myocardial infarction, and stroke in high risk patients. BMJ 2002;324:71-86.

22 Mehta SR, Bassand JP, Chrolavicius S et al. Effects of clopidogrel in addition to aspirin in patients with acute coronary syndromes without ST-segment elevation. N Engl J Med 2001;345:494-502.

23 Wallentin L, Becker RC, Budaj A et al. Ticagrelor versus clopidogrel in patients with acute coronary syndromes. N Engl J Med 2009;361:1045-57.

24 Wiviott SD, Braunwald E, McCabe $\mathrm{CH}$ et al. Prasugrel versus clopidogrel in patients with acute coronary syndromes. N Engl J Med 2007:357:2001-15.

25 Dworeck C, Redfors B, Angeras O et al. Association of pretreatment with ${\mathrm{P} 2 \mathrm{Y}_{12}}_{12}$ antagonists preceding percutaneous coronary intervention in non-ST-segment elevation acute coronary syndromes with outcomes. JAMA Netw Open 2020;3:e2018735. 
26 Schüpke S, Neumann F-J, Menichelli M et al. Ticagrelor or prasugrel in patients with acute coronary syndromes. N Engl J Med 2019;381:1524-34.

27 Subherwal S, Bach RG, Chen AY et al. Baseline risk of major bleeding in non-ST-segment-elevation myocardial infarction: the CRUSADE (Can Rapid risk stratification of Unstable angina patients Suppress ADverse outcomes with Early implementation of the ACC/ AHA Guidelines) Bleeding Score. Circulation 2009;119:1873-82.

28 Urban P, Mehran R, Colleran R et al. Defining high bleeding risk in patients undergoing percutaneous coronary intervention: a consensus document from the Academic Research Consortium for High Bleeding Risk. Eur Heart J 2019;40:2632-53.

Address for correspondence: Dr Ramesh Nadarajah, Leeds Institute of Cardiovascular and Metabolic Medicine, 6 Clarendon Way, Leeds LS2 9DA, UK.

Email: r.nadarajah@leeds.ac.uk

Twitter: @RameshNadaraja2 